\title{
CRANIO-CARPO-TARSAL DYSTROPHY
}

\author{
An Undescribed Congenital Malformation
}

\begin{abstract}
BY
E. A. FREEMAN, F.R.C.S., Hon. Orthopaedic Surgeon, and

J. H. SHELDON, M.D., F.R.C.P., Hon. Physician to the Royal Hospital, Wolverhampton

The two children who form the subject of this paper were shown at the Section for the Study of Disease in Children of the Royal Society of Medicine on March 25, 1938, when it was considered that they were examples of a previously undescribed condition (Freeman and Sheldon, 1938). Both cases exemplify a number of similar features, and as there can be little doubt that these go together to form one disease entity, a full account is offered in view of the fact that this appears to be its first description.
\end{abstract}

\section{Clinical Records}

Case 1. A male aged two years nine months, with no relevant family history or consanguinity of parents, was an elder child, the second baby being normal. The patient was a seven-and-one-half-months baby, born by normal labour without instruments. The weight at birth was $3 \frac{1}{2} \mathrm{lb}$. including clothes. His appearance at birth caused such consternation 'that he was taken downstairs and shown to the grandmother, and then put out to die.' The legs were born fully flexed on the trunk in a 'Buddha' position, and at the back of the head was a lump described as "jelly-blood' which disappeared naturally in some three or four days. The nature of this is uncertain. Its description is not that of a cephalhaematoma, and neither by $x$-rays nor clinically is there now any evidence of abnormality in the posterior part of the head. The face was abnormal. The lower forehead and eyebrows were occupied by a soft but prominent transverse swelling and the eyes were buried so deeply that they were hardly visible. The nose could barely be recognized and the mouth was small. In addition to these features, the hands showed ulnar deviation and the feet a severe degree of talipes equino-varus. In spite of the immediate reaction to his appearance in the world, efforts were made to rear him, which were all the more difficult in that for some days he was unable to suckle owing to difficulty in swallowing. He was fed with drops of fluid from an eyedropper, since more than one drop at a time 'stuck in his throat.' Eventually swallowing became normal, and he was reared on a dried milk. On account of the deformity in the feet he was sent to the Orthopaedic Department of the Royal Hospital, Wolverhampton, when a fortnight old. He continued to receive treatment for the feet until May, 1937 (age twenty-three months), when he was admitted under Mr. R. Milnes Walker, F.R.C.S., Hon. Surgeon to the 
Royal Hospital, Wolverhampton, on account of a swelling in the right loin. At operation this was found to be due to torsion of an imperfectly descended testicle, the organ being removed as it was partly gangrenous. He made a

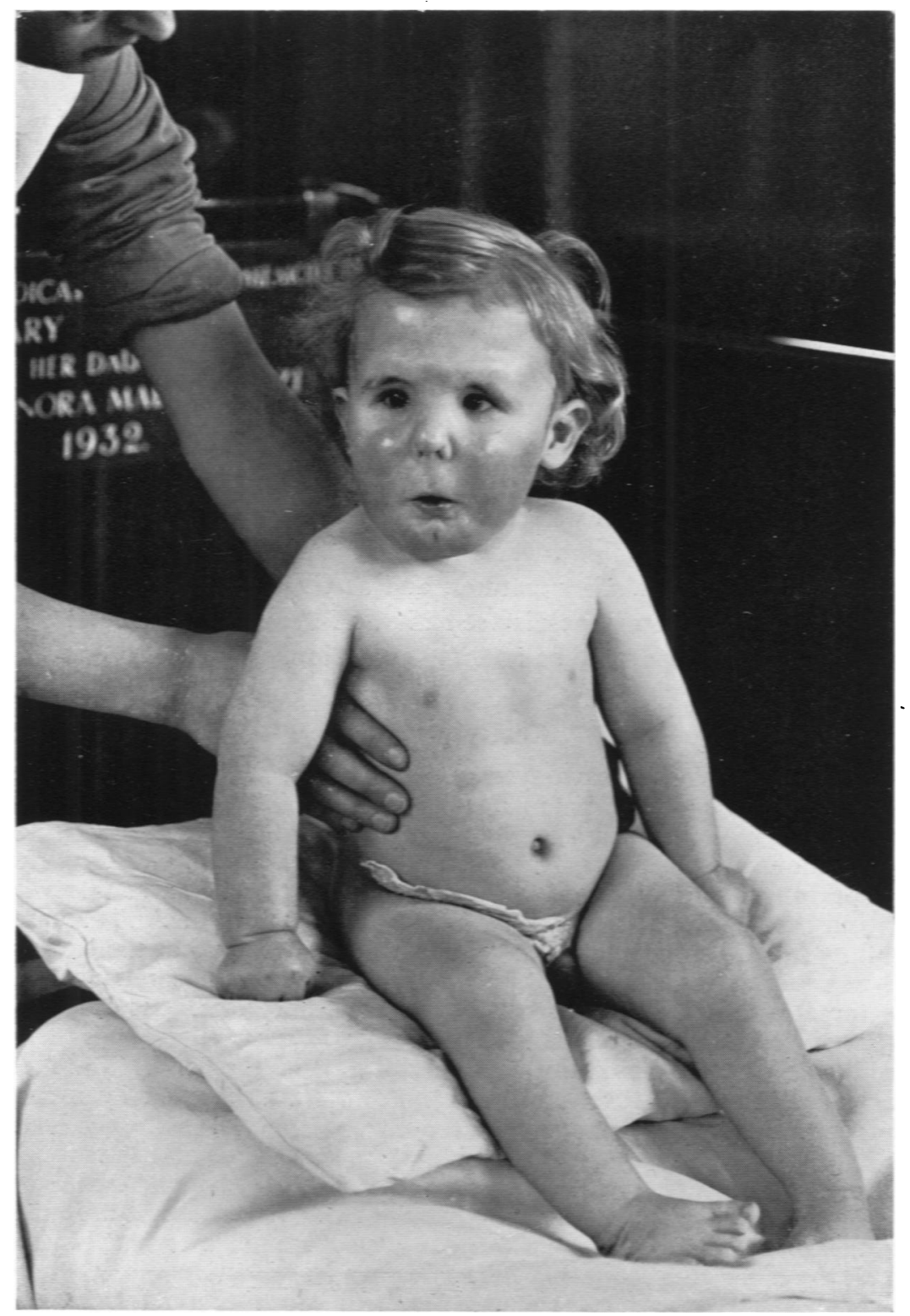

FIG. 1.

good recovery from the operation, but before leaving hospital he developed a pneumonia followed by empyema, surgical drainage of which led to a complete recovery. Since then he has been receiving further orthopaedic treatment, and his general health has remained good. 
Present Condition.-Length : 2 feet 8 inches. Weight : $27 \frac{1}{2} \mathrm{lb}$. Routine clinical examination reveals no abnormalities beyond those detailed below. Mentality is fair. He is able to talk, to frame short sentences and can repeat simple nursery rhymes. He has an unusually good 'musical' ear. During the first eighteen months of life the mentality was backward, but he has been catching up since, so that he is now not far behind the normal for his age.

FACE. - This is the most obvious feature and is shown in fig. 1. Across the lower forehead just above the eyebrows is a soft, subcutaneous swelling, which is shiny in reflected light and moves with the facial muscles. Similar

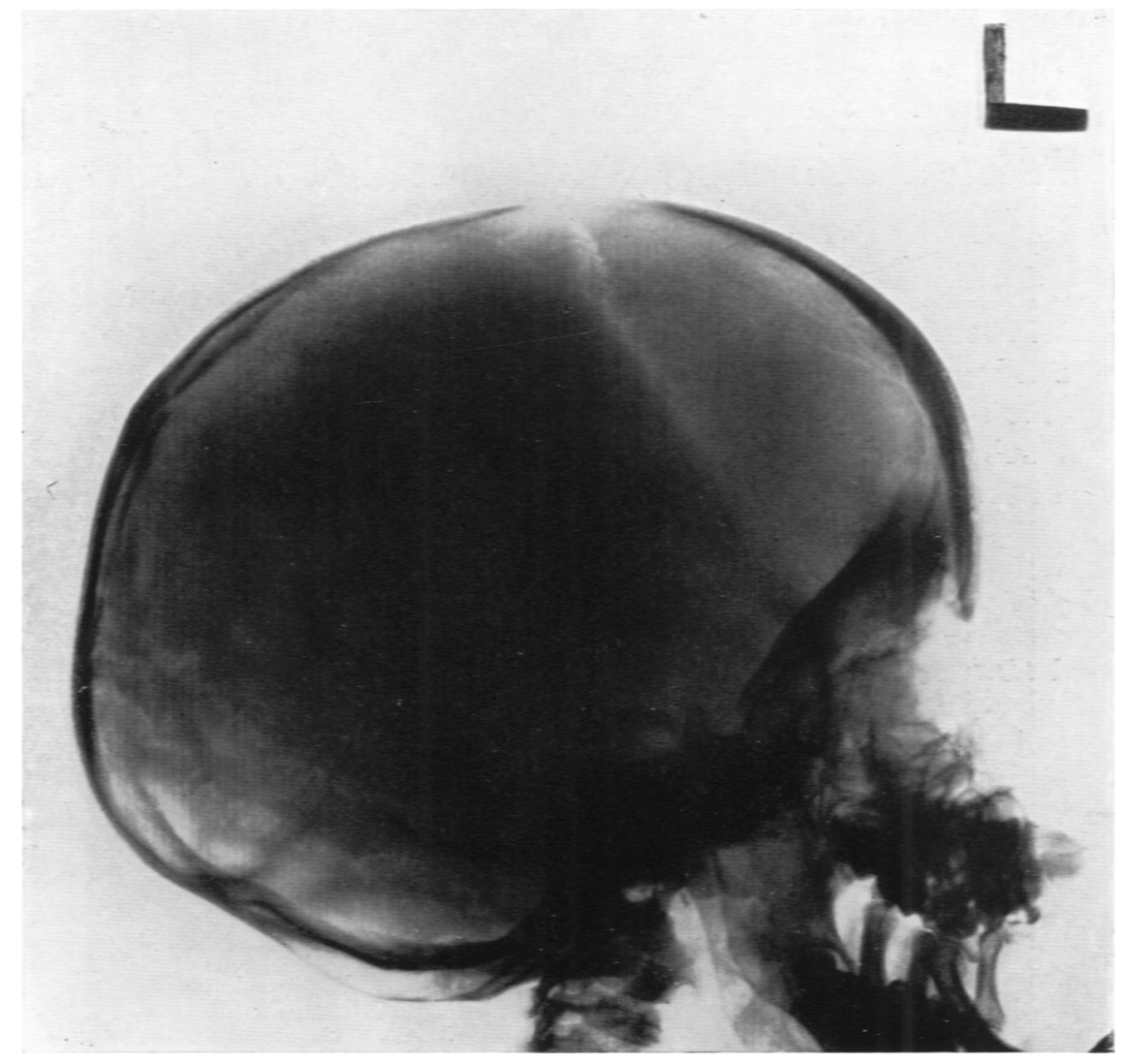

Fig. 2.-X-ray of skull of case 1. (The lower jaw is obscured by the nurse's hand.)

subcutaneous ' bosses' are present on each side of the upper forehead and are clearly visible in the photograph. The eyes are deeply sunken, and there is an internal strabismus of the left eye. The eyes appear to be more widely separated than normal. The bridge of the nose is broad and disproportionately big in comparison with its lower part, which is small and narrow and does not rise above the face to a normal extent. This feature was more marked at birth. The nasal openings are small. Both cheeks are abnormally fat, rosy and shiny. (This is well seen in the photograph.) The distance from the nose to the upper lip is abnormally great, and at the first view of the child this is probably the most striking feature. The mouth is small in proportion to the 
rest of the face, and the upper lip appears smaller than the lower. The tongue is small. That the mouth really is small is seen from the fact that at the age of two years it was almost impossible to introduce a wooden spatula, and a

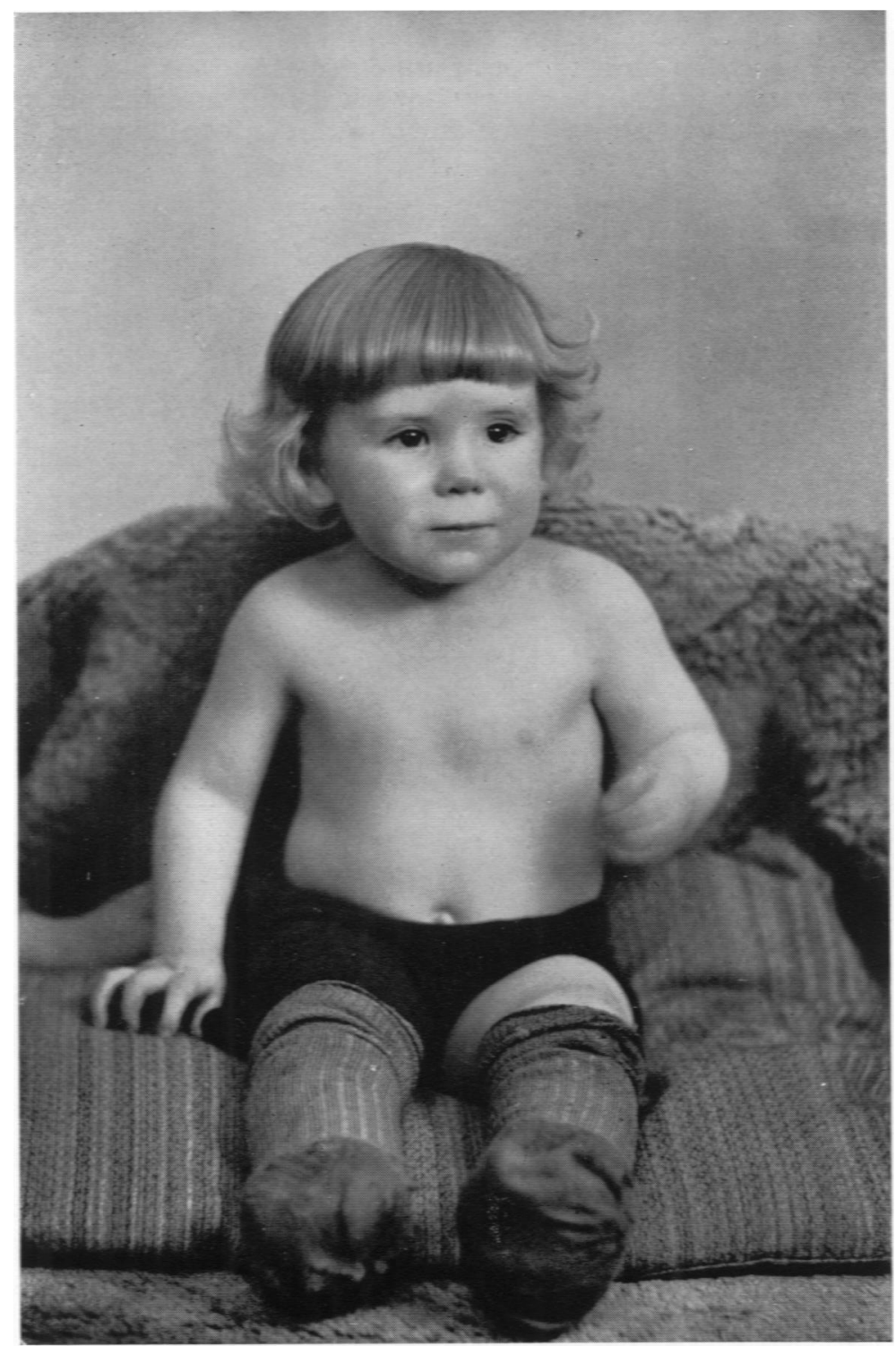

Fig. 3.

tea-spoon could not be admitted. The palate is highly arched, but the teeth are normal except for caries. There is an unusually well-marked dimple on the mid-point of the chin. Taken all in all, the part of the face below the eyes appears too small in proportion to the forehead. 
An x-ray photograph of the skull is shown in fig. 2. On comparison with the normal skull there are three abnormalities : (1) The floor of the skull in the anterior fossa slopes steeply upwards towards the frontal bone. (2) There appears to be a partial failure of union between the frontal bone and the floor of the skull, so that there is a free prolongation downwards of the lower end of the frontal bone. (3) In relation to the total antero-posterior length of the skull, the part occupied by the nasal and facial bones appears to be less than normal. Antero-posterior x-ray examination of the skull did not reveal any abnormal features.

HANDS.-The hands show ulnar deviation, but no associated bony

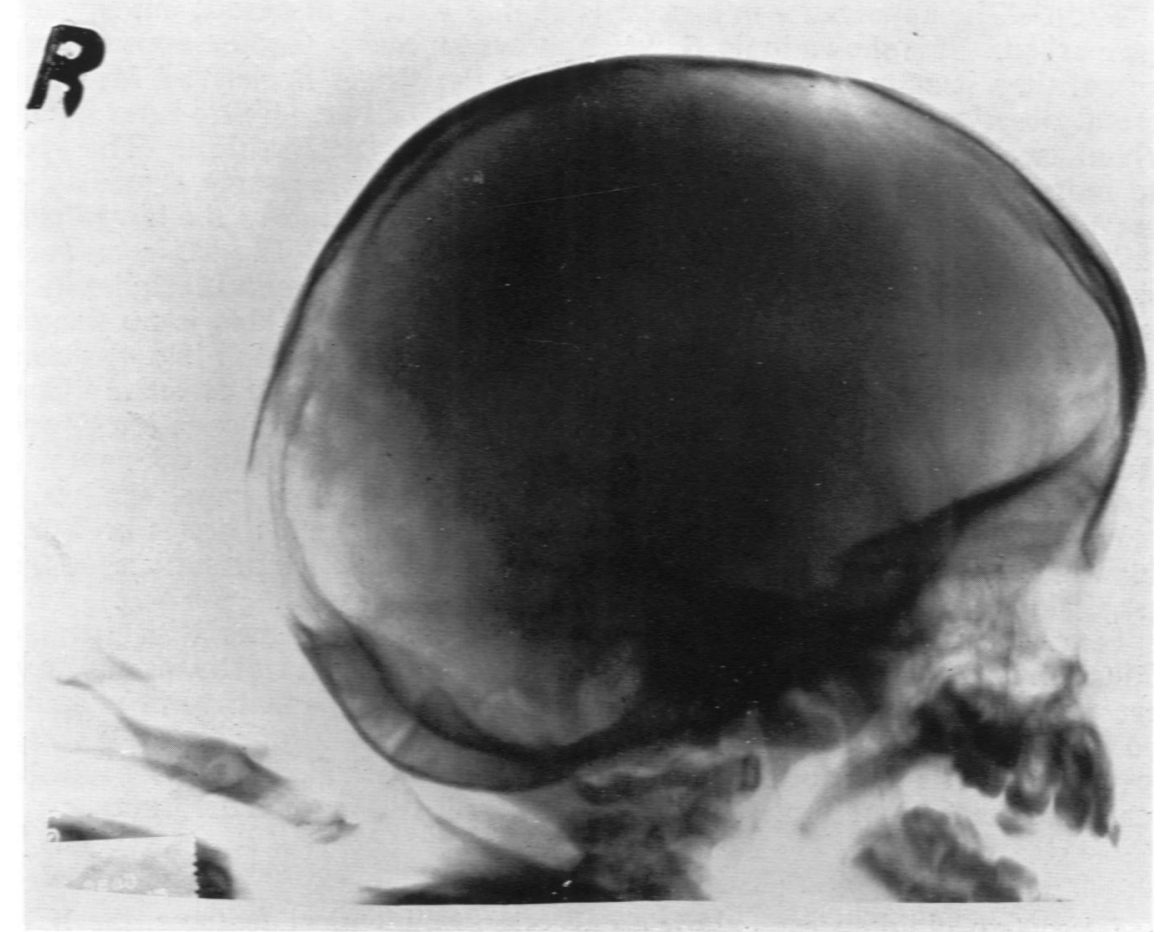

FiG. 4.-X-ray of skull of case 2.

abnormality can be seen on $x$-ray examination. Without treatment there has been considerable improvement in the function of the hands since birth. The hands are covered with a firm fat, like that on the face. On the flexor surfaces of the first phalanges of both hands there is a considerable degree of horny thickening of the skin and subcutaneous tissues.

FEET.- The feet show an extremely severe bilateral talipes equino-varus, with no unusual features. They have received a good deal of treatment with disappointing results. Correction was first attempted by means of pads and strapping by Brockman's method. Little progress was made, and plaster correction was resorted to, again without success. More recently an attempt 
has been made to free the scaphoid from its attachments on the inner side, but further bony correction will probably be necessary at a later date.

Case 2. A female, age nineteen months, was an only child, with no relevant family history and no consanguinity of parents. She was an eight months' child, born by normal labour. The weight at birth was $4 \frac{1}{2} \mathrm{lb}$. It was immediately obvious that the hands and feet were deformed, but nothing unusual was noticed in the face except that the mouth was small. She was able to suckle and there was no difficulty in swallowing. She was breast-fed for fourteen days, and then given a dried milk. As with the previous case, she was brought at an early age to the Orthopaedic Department of the Royal Hospital on account of the feet.

Present Condition.-Length : 2 feet 7 inches. Weight : $32 \mathrm{lb}$. Routine clinical examination revealed no abnormalities beyond those detailed below. Mentality is fair - she can say simple words, but not sentences.

FACE.-The appearance in general is similar to that of case 1 , but is much less marked. It is shown in fig. 3, which, however, makes the face appear more normal than it actually does to the eye. At the Royal Society of Medicine several observers took the children for brother and sister. There is no supraorbital swelling and no subcutaneous bosses over the forehead. The eyes are more deeply sunken than normal, though not to the same extent as in case 1. The upper part of the nose is broad in proportion to the lower half, which does not rise out of the face to a normal extent. The space between the lower end of the nose and the mouth is big, and the mouth is smaller than normal though it is not suggested by the photograph. There is no dimple over the symphysis of the chin. X-ray examination of the skull (fig. 4) shows the same general appearance as in case 1 . There is the same defective union between the frontal bone and the floor of the skull, with a prolongation downwards of the lower end of the frontal bone. The floor of the skull does not rise up so sharply as in case 1 , nor do the facial and nasal bones appear so shallow.

HANDS. - These show ulnar deviation as in case 1 , and there is no bony abnormality to be seen by $x$-ray examination. On the flexor surface of the first phalanges there is the same horny thickening as in case 1 .

FEET.-These show a severe bilateral talipes equino-varus with extreme adduction and inversion. As with the previous case, much treatment has been necessary. Correction was first attempted by Brockman's method of padding and strapping, and later plaster correction was used, also without success. Operation was then performed, the scaphoid being freed along its inner attachments and the feet put in plaster again, but there is still marked deformity.

\section{Discussion}

These two children each show a collection of identical features, the only differences being in their sex and in the fact that the facial appearance is much more normal in the second case. These features are :

(1) They are both first children.

(2) Premature birth ( $7 \frac{1}{2}$ and 8 months), to which the small weight is attributable.

(3) An abnormal facies.

(4) An abnormal x-ray appearance of the anterior end of the skull.

(5) Ulnar deviation of the hands with thickening of the skin and subcutaneous tissues over the flexor surface of the first phalanx of the fingers.

(6) Severe talipes equino-varus. 
The simultaneous appearance of these various features in two children leaves little room for doubt that they hang together as a single disease entity, the most probable explanation of which is that it is due in each case to a similar genetic abnormality. Although they both come from the same town, they do not appear to be related, although it has not been possible to take the enquiries very far back. The grandparents were not related, and of their sixteen greatgrandparents only twelve can be identified, but these were not related. Inasmuch as the condition appears to be an undescribed one, the name of 'craniocarpo-tarsal dystrophy' has been provisionally applied to it.

Thanks are due to Dr. G. E. Dyas, Hon. Radiologist to the Royal Hospital, Wolverhampton, for his help with the $\mathrm{x}$-ray examinations.

\section{REFERENCE}

Freeman, E. A., and Sheldon, J. H. (1938). Proc. roy. Soc. Med., 31, 1116. 